Internal Report

DESY M-83-21

September 1983

BEAM INSTABILITIES AND COMPUTER SIMULATIONS

by

A. Piwinski 
DESY behält sich alle Rechte für den Fall der Schutzrechtserteilung und für die wirtschaftliche Verwertung der in diesem Bericht enthaltenen Informationen vor.

DESY reserves all rights for commercial use of information inciuded in this report, especially in case of filing application for or grant of patents.

"Die Verantwortung für den Inhalt dieses Internen Berichtes liegt ausschließlich beim Verfasser" 
DESY M-83-21

September 1983

\section{BEAM INSTABILITIES AND COMPUTER SIMULATIONS}

A. Piwinski

Invited paper presented at the Europhysics Conference on Computing in Accelerator Design and Operation Berlin (West), September 20.-23.1983 
BEAM INSTABILITIES AND COMPUTER SIMULATIONS

A. Piwinski

Deutsches Elektronen-Synchrotron. DESY

Notkestraße 85, D-2000 Hamburg 52, W. Germany

\section{Introduction}

The most important instability which limits the luminosity in existing electron-positron storage rings is caused by beam-beam interaction. But. also synchro-betatron resonances are a serious problem and can limit the luminosity. The beam-beam interaction leads to a blow-up of the beams which reduces the luminosity and the life time of the colliding bunches. Synchro-betatron resonances or satellite resonances occur if the relation

$$
k Q_{x}+l_{z}+m Q_{S}=n
$$

is satisfied where $k, l, m$ and $n$ are integers and $Q_{X}, Q_{z}$ and $Q_{S}$ are the betatron and synchrotron frequencies in units of the revolution frequency. With increasing bunch current the number and the width of the satellite resonances increase such that finally the currents are limited.

These satellite resonances are mainly excited by dispersions in the accelerating cavities and by transverse fields with a longitudinal gradient in the cavities. Satellite resonances due to the chromaticity are usually prevented by sextupoles. Dispersions in the cavities cannot be supressed completely. Especially during energy ramping when the optics is changed and the orbit is shifted by small amounts, spurious dispersions are produced. Transverse fields with a longitudinal gradient are generated when passing the cavities off-axis which is difficult to avoid during energy ramping. An analytical treatment of satellite resonances is difficult. Especially the increase or decrease of the oscillation amplitudes during many revolutions cannot be calculated. Computer simulations, however, can show the general behaviour of the particles on such a resonance.

Satellite resonances can also be excited by the beam-beam interaction when the beams cross at an angle or when the dispersion at the interaction point is not zero. In that case many satellites of nonlinear betatron resonances appear which were found by computer simulations and seen in the storage ring DORIS I, where the beams crossed at an angle. This mechanism might also become important for the new ep-project HERA。

The blow-up of the beams at head-on collision is caused by the strong nonlinearities of the space charge forces. Many resonances can be excited, especially coupling resonances of horizontal and vertical betatron oscillations such that the oscillation energies between the horizontal and vertical plane are exchanged. The beam height, which is usually much smaller than the beam width, is then increased drastically whereas the width remains nearly constant. An analytical treatment of this effect is, at the moment, not yet possible. Computer simulations are, therefore, the best way to investigate the dependence of the blow-up on various machine parameters and to find cures to suppress the blow-up as far as possible. 


\section{Synchro-betatron resonances}

\subsection{Excitation caused by dispersions in a cavity}

For each particle with an arbitrary energy deviation exists a closed orbit that can be described as the product of the dispersion D times the relative momentum deviation or approximately, the relative energy deviation:

$$
x_{c_{0} .0}(l)=D_{x}(l) \frac{\Delta p}{P} \approx D_{x}(l) \frac{\Delta E}{E}
$$

Around this closed orbit the particles perform betatron oscillations. The dispersion can be horizontal or vertical and we consider horizontal or vertical satellite resonances. In the cavity the energy is changed and therefore the closed orbit is shifted. Since the total coordinates $x$ and $x^{\prime}$ cannot be changed in such a short interval the betatron coordinates are changed:

$$
\begin{aligned}
\delta x & =-D_{x} \frac{\delta E}{E} \\
\delta x^{\prime} & =-D_{x}^{\prime} \frac{\delta E}{E} \\
\delta E & =\operatorname{eU}(\sin (\psi+2 \pi s / \lambda)-\sin \psi)
\end{aligned}
$$

with $\mathrm{e}=$ elementary charge, $U$ = cavity voltage, $\psi=$ synchronous phase, $s$ = longitudinal position, $\lambda=$ wave length of the voltage.

We take into account only that part of the eneray change which varies with 'the synchrotron frequency. The other constant part which replaces the radiation losses does not play a role for a resonance. The longitudinal position is changed in the curved sections of the machine. The change of $s$ per revolution is given by

$$
\delta s=\oint \frac{1}{\rho}\left(x+D_{x} \frac{\Delta E}{E}\right) d l=A_{1} x+A_{2} x^{\prime}-\alpha_{M} c \frac{\Delta E}{E}
$$

with

$$
\begin{aligned}
& A_{1}=-\frac{1}{B_{x}}\left(D_{x} \sin \mu_{x}-F_{x}\left(1-\cos \mu_{x}\right)-A_{2} \alpha_{x}\right. \\
& A_{2}=-D_{x}\left(1-\cos \mu_{x}\right)-F_{x} \sin \mu_{x} \\
& F_{x}=\alpha_{x} D_{x}+B_{x} D_{x}^{\prime} \quad \alpha_{x}=-B_{x}^{\prime} / 2
\end{aligned}
$$

$\alpha_{M}$ is the momentum compaction factor and $C$ is the circumference, $\rho$ is the radius of curvature and $\mu_{x}$ is the betatron phase advance per revolution.

$x$ and $x^{\prime}$ are the betatron coordinates at the beginning of the revolution.

A simulation of the coupled synchrotron and betatron oscillations on a digital computer has been done ${ }^{1)}$ for small currents taking into account the natural nonlinearity of the synchrotron oscillation (Eq. (5)). The simulation has the following steps: In the cavity the longitudinal coordinate $s$ of the synchrotron oscillation remains constant whereas the energy is changed according to Eq. (5). The change of the betatron coordinates is given by Eqs. (3) and (4). In the arc the change of $s$ is given by Eq. (6) whereas the energy deviation is constant. The betatron coordinates $x$ and $x^{\prime}$ are transformed with

$$
M=\left(\begin{array}{ll}
\cos \mu_{x}+\alpha_{x} \sin \mu_{x} & \beta_{x} \sin \mu_{x} \\
-\left(1+\alpha_{x}^{2}\right) \sin \mu_{x} / B_{x} & \cos \mu_{x}-\alpha_{x} \sin \mu_{x}
\end{array}\right)
$$

Fig. I shows the typical behaviour of the amplitudes during 1200 revolutions. Fig. la shows the betatron and synchrotron amplitudes for a sum resonance where always one amplitude increases while the other one decreases. Fig. lb shows that on a difference resonance both amplitudes increase or decrease at the same time. In this case the beat is larger than in the first case. 


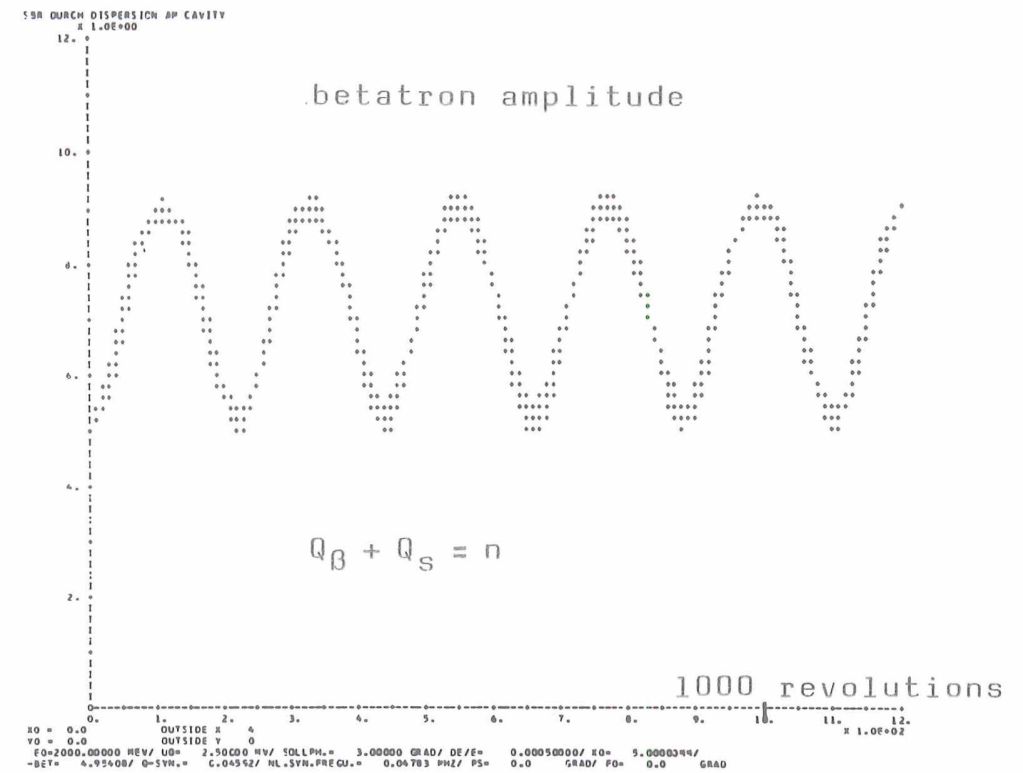

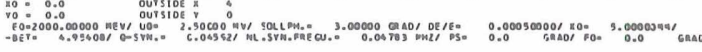

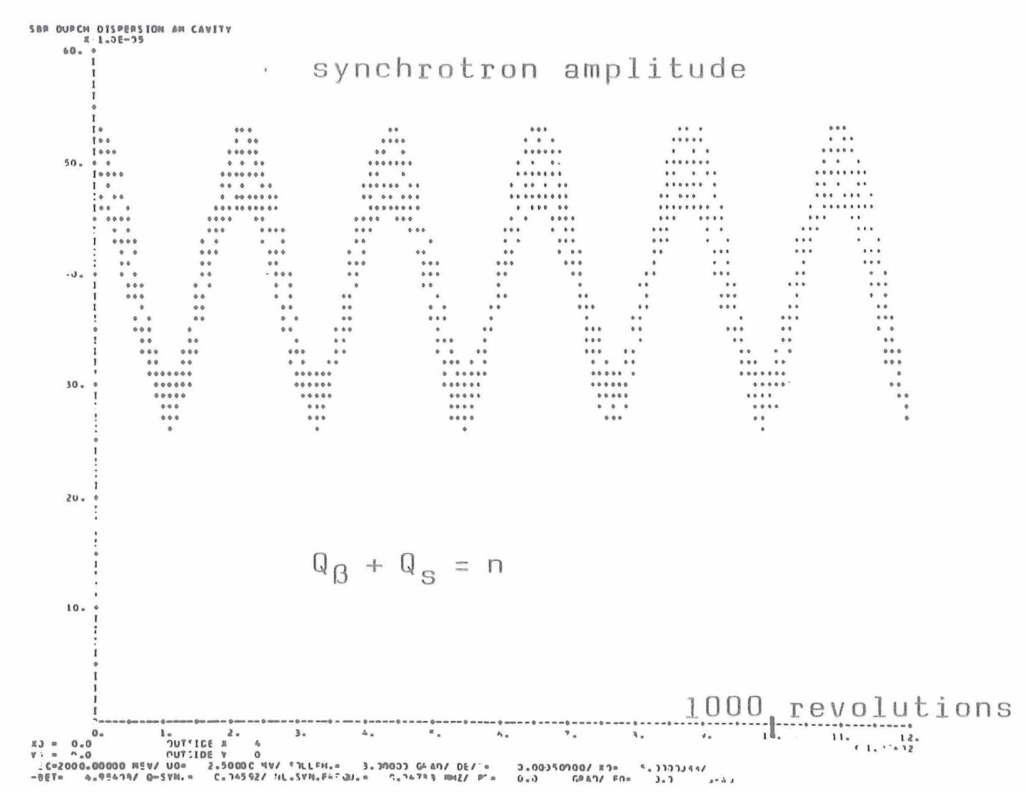

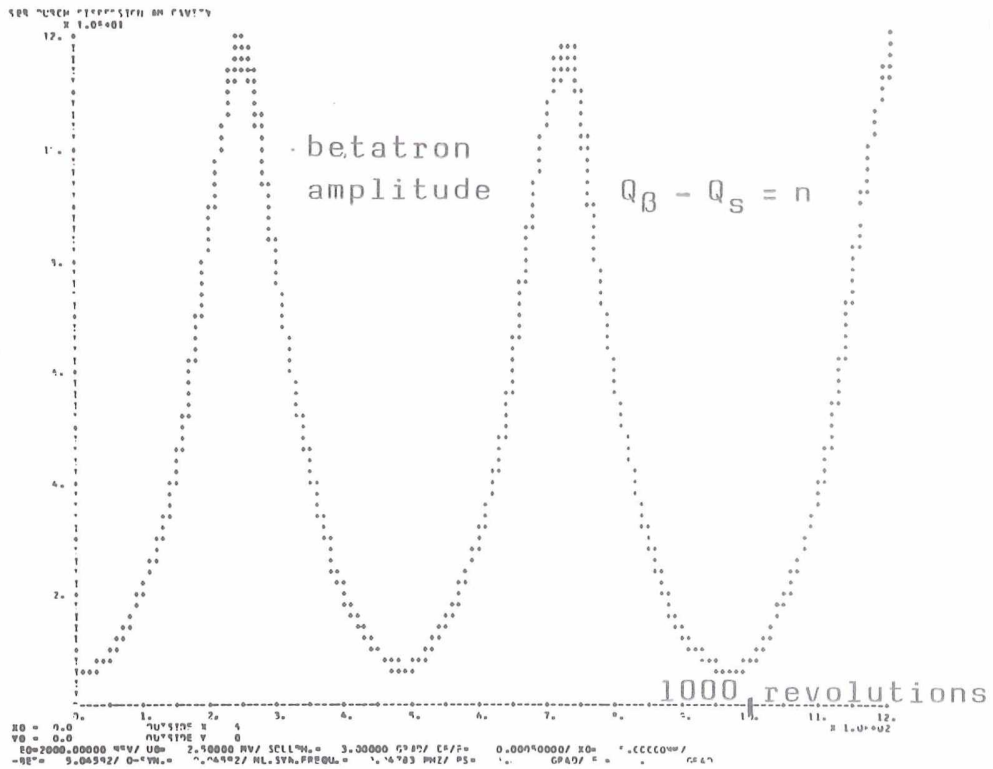

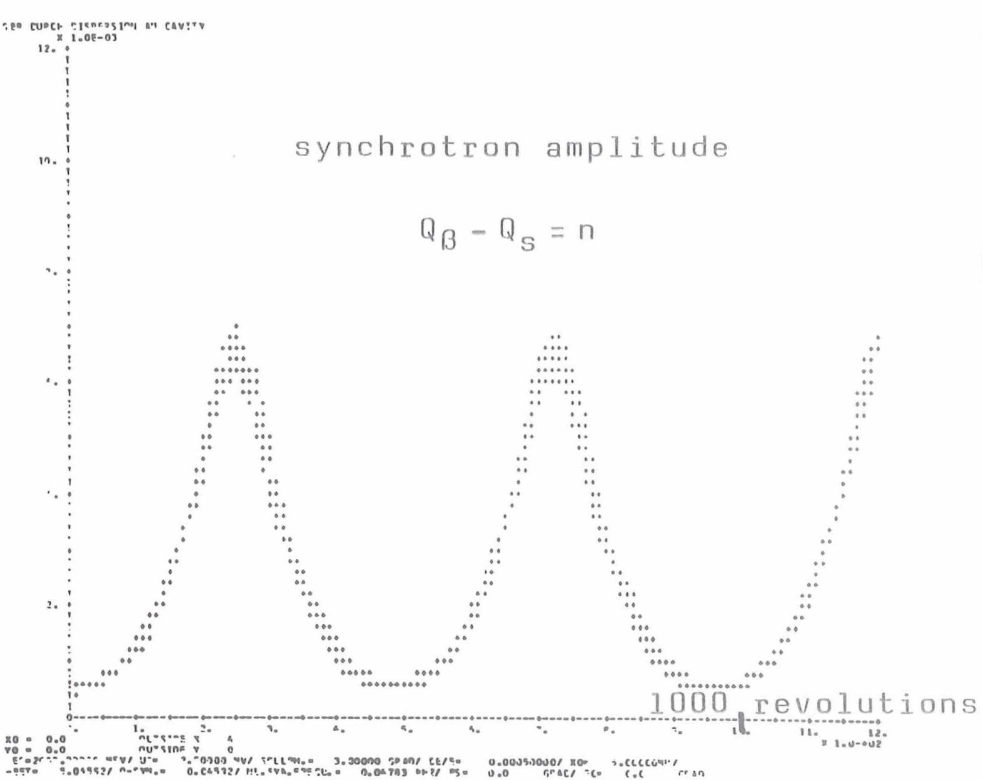

Fig. 1: Betatron and synchrotron amplitudes in arbitrary units as a function of time

a) on a sum resonance

b) on a difference resonance 


\subsection{Excitation caused by transverse fields with a longitudinal gradient}

Transverse fields which vary with the longitudinal position of a particle in a bunch can be caused by the accelerating voltage due to an angle between the cavity axis and the closed orbit or due to asymmetries in the cavities ${ }^{2}$ ) and by the bunch itself due to off-center passage or due to cavity asymmetries ${ }^{3}$ ). The vertical kick of a particle produced by transverse fields is given by

$$
\delta z^{\prime}=\frac{e}{p} \int\left(E_{z}+v B_{x}\right) d t
$$

where $E_{Z}$ and $B_{X}$ are the electric and magnetic fields and $v$ is the particle velocity. The integral is taken along the path of the particle between two limits where the fields vanish. The region of the fields is assumed to be short as compared to the wave length of the betatron oscillation.

We consider that part of $\delta z^{\prime}$ which varies linearly with the longitudinal position $s$ of a particle in the bunch and obtain with Maxwell's equations

$$
\delta z^{\prime}=s \frac{e}{p} \int\left(\frac{\partial E_{z}}{\partial s}+v \frac{\partial B_{x}}{\partial s}\right) d t=s \frac{e}{p} \int \frac{\partial E_{s}}{\partial z} d t=A s
$$

If the transverse fields have a longitudinal gradient the longitudinal field must have a transverse gradient, and that means that the betatron oscillation influences the synchrotron oscillation. That part of the energy change which varies linearly with the transverse position of a particle in the bunch is given by ${ }^{4}$ )

$$
\frac{\delta E}{E}=z \frac{e}{E} \int \frac{\partial E_{S}}{\partial z} v d t=\frac{v^{2}}{c^{2}} A z \approx A z
$$

A comparison of Eqs. (3), (4) and (9) shows that the increase of betatron oscillation depends in the same way on the synchrotron oscillation, namely on $s$. The two mechanisms, caused by a dispersion and by transverse fields, can at least for slowly changing synchrotron amplitude compensate each other. This is also possible for higher harmonics of the synchrotron frequency produced by nonlinearities. Then the compensation depends on the amplitude of the synchrotron oscillation and on the bunch current.

\subsection{Excitation caused by the beam-beam interaction at a crossing angle}

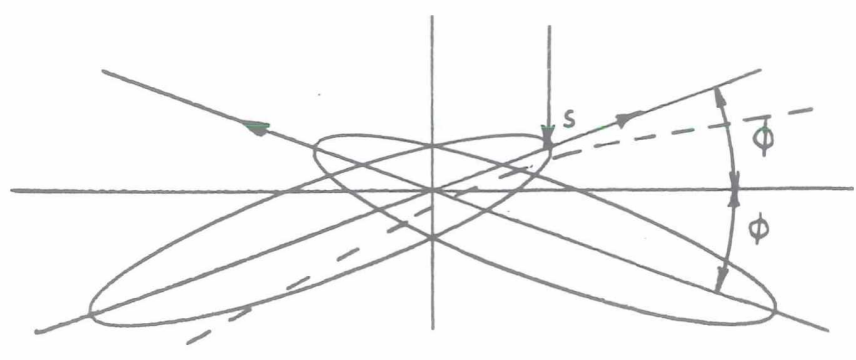

Fig. 2: Orbit distortion due to a crossing angle

We consider a particle which has a distance $s$ from the center of its own bunch. This particle does not pass through the center of the other bunch and hence gets a vertical kick. The kick has the direction indicated in Fig. 2 if we assume particles with different signs as electrons and positrons. Because of this kick the axis of the bunch cannot be the closed orbit for the considered particle, but the closed orbit must look like the dotted line.

The kick, i.e. the change of the vertical betatron angle $z^{\prime}$ can be written in the form

$$
\delta z^{\prime}=f(z+s \Phi)
$$

where $f$ describes the space charge forces as a function of the vertical position $z$ and the longitudinal position s. 
The synchrotron oscillation is also influenced by the betatron oscillation since the energy is changed for a particle that crosses the bunch at an angle. The energy change is understandable, if one takes into account that the kick or momentum change is vertical. One can then decompose the change of the momentum and obtains a longitudinal component $p_{s}$ which is given by $\Phi p_{z}$. The energy change is then

$$
\frac{\delta E}{E}=\frac{\delta P}{P}=\frac{\delta P_{S}}{P}=\Phi \frac{\delta P_{z}}{P}=\Phi \delta z^{\prime}=\Phi f(z+s \Phi)
$$

If the effective cross section is circular as in DORIS I ( $\sigma_{\text {zef }} \approx \sigma_{x}=\sigma$ ) an exact expression for the function $f(E q .(11)$ ) can be obtained:

$$
f(u)=\frac{2 r_{e} N_{b}}{\gamma u} \quad\left(e^{-\frac{u^{2}}{2 \sigma^{2}}}-1\right)
$$

with $r_{e}=$ electron radius, $N_{b}=$ number of particles per bunch, $\gamma=$ particle energy divided by its rest energy, $\sigma=$ standard deviation of the Gaussian-like particle distribution.

A simulation. of the betatron and synchrotron oscillation including the nonlinear coupling (Eq. (13)) has been done on a digital computer. Between the interaction points the oscillations were transformed linearly. Fig. 3 shows the ratio of the maximum to the minimum betatron amplitude during 2000. revolutions for $\Phi=12 \mathrm{mrad}$, $N_{b}=8 \cdot 10^{9}, \gamma=3500, \sigma=.23 \mathrm{~mm}, Q_{S}=.034, B_{Z}^{*}=1 \mathrm{~m}$. More than $1000 Q_{B}$-vailues between 6.02 and 6.48 are investigated but only those resonances are shown which reach an increase of more than $50 \%$ of their initial amplitude. Measurements with the storage ring DORIS I have proved that exactly at these frequencies the life time of the bunch dropped to a few seconds ${ }^{5}$ ).

Analytical investigations and the latest measurements with DORIS II have shown that also a dispersion at the interaction point can excite similar synchro-betatron resonances ${ }^{5,6}$ ).
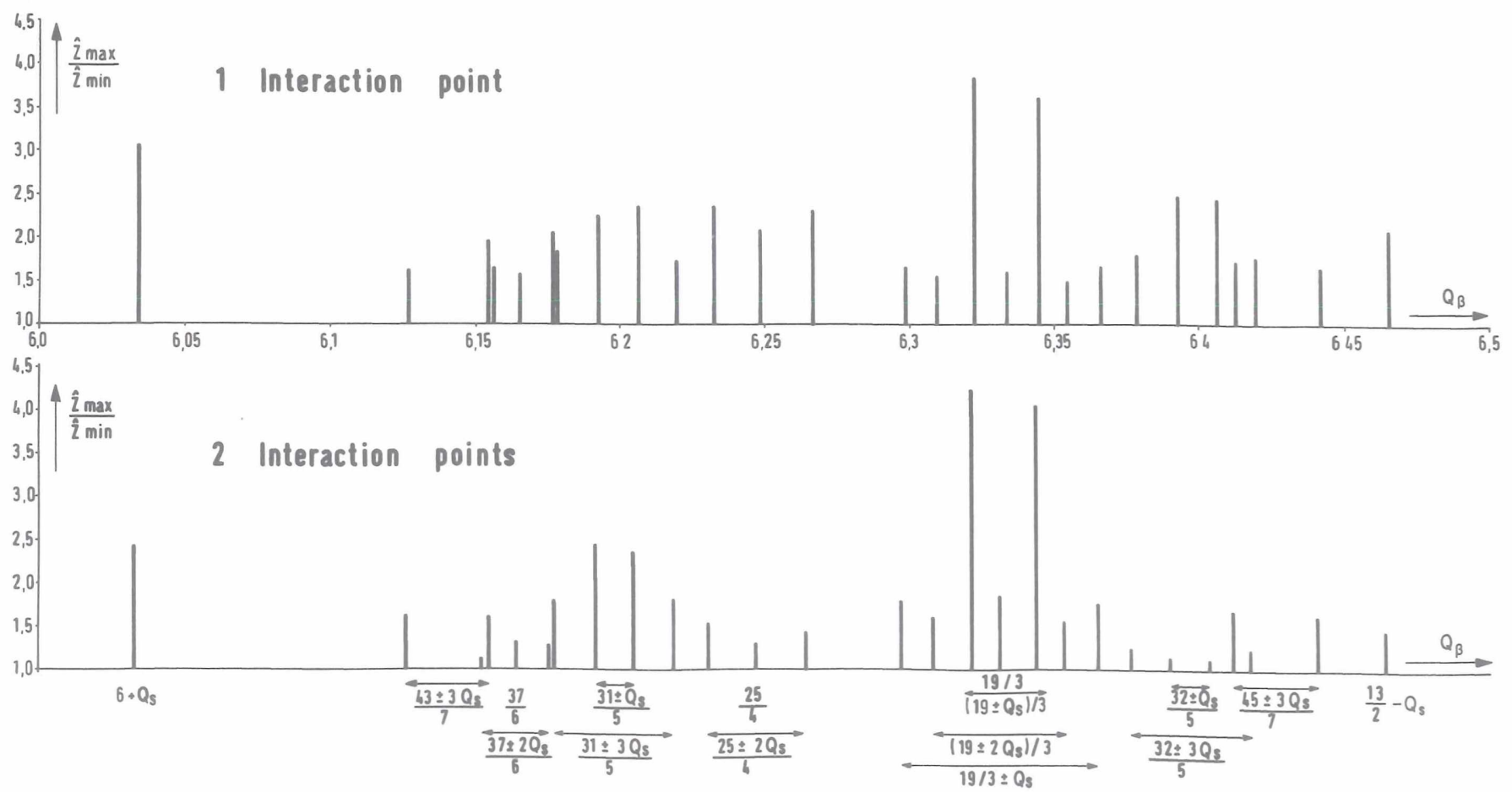

Fig. 3: Maximum betatron amplitude as a function of the betatron frequency 


\section{Beam-beam interaction}

In simulating the beam-beam interaction on a digital computer, significant progress was made in $\left.1980^{7,8}, 9,10\right)$. Since that time, many simulations were done by several authors, and a better understanding of the mechanism of the blow-up was achieved. In the following the simulations for PETRA are briefly described.

The space charge forces are exactly calculated for a relativistic bunch with a Gaussian particle distribution. The change of the betatron angles $x^{\prime}$ and $z^{\prime}$ at each interaction point is then given by

$$
\left|\begin{array}{c}
\Delta x^{\prime} \\
\Delta z^{\prime}
\end{array}\right|=-\frac{2 \pi}{1-V} \int_{0}^{1-V^{2}} \exp \left\{- \text { as }-\frac{\text { bs }}{1-s}\right\} \quad\left|\begin{array}{l}
x \xi_{x} / \beta_{x o} \\
\frac{z \xi_{z} / \beta_{z 0}}{1-s}
\end{array}\right| \frac{d s}{\sqrt{1-s}}
$$

with $\quad V=\sigma_{z} / \sigma_{x} ; a=\frac{1}{2} \frac{x^{2}}{\sigma_{x}^{2}-\sigma_{z}^{2}} ; b=\frac{1}{2} \frac{z^{2}}{\sigma_{x}^{2}-\sigma_{z}^{2}} ; \xi_{x, z}=\frac{r_{e} N_{b} \beta_{x, z}^{*}}{2 \pi r \sigma_{x, z}\left(\sigma_{x}+\sigma_{z}\right)}$

The kicks were calculated for a two-dimensional grid of points and then interpolated quadratically for calculating the transverse kick of a particle at each passage. The longitudinal motion of the interaction point seen by a particle due to its synchrotron oscillation is always taken into account. Between the interaction points the horizontal and vertical betatron oscillation and the synchrotron oscillation are transformed linearly. The radiation damping is included. The quantum fluctuation is simulated by applying random kicks on all three modes. of oscillation. The motion of particles is observed over several damping times, i.e. over a large number of revolutions. Both cases, "weak-strong" and "strong-strong" were investigated.

As an example Fig. 4 shows the vertical motion of a single particle in the phase diagram. The horizontal axis gives the position and the vertical axis the angle of the vertical betatron oscillation at a symmetry point of the machine for each revolution. The vertical amplitude of the particle starting with zero amplitudes remains within one or two standard deviations of the Gaussian distribution of the opposing bunch during the first 8000 revolutions (a). Then its amplitude increases rapidly due to quantum fluctuation and the nonlinearity of the space charge forces, and it moves into a third order resonance where the phase advance is about $2 \pi / 3+2 \pi \times$ integer (b). After about three quarters of a damping time it comes out of resonance and leaves the three fixed points, but is immediately captured by other three fixed points of the third order resonance (c) which are a mirror image and equivalent to the first three points (without optical asymmetries). After about 600 revolutions the particle leaves the third order resonance (d).

A similar behaviour can be observed for several resonances. More often, however, coupling resonances between horizontal and vertical betatron oscillations appear. These resonarices can be found by counting the betatron oscillations and by observing the variation of the amplitudes. In all cases the particles usually do not stay longer than a damping time on a resonance. Due to quantum fluctuation and dam ping they can leave the resonance and can then be captured by another resonance.

The computer simulations have shown that small disturbances of the ideal machine increase the number and strength of the resonances which can be excited. Those disturbances are small differences in betatron phase advance between the interaction points and spurious dispersions at the interaction points. Thus machine imperfections enlarge the blow-up of the beams and become more important with increasing number of interaction points. 


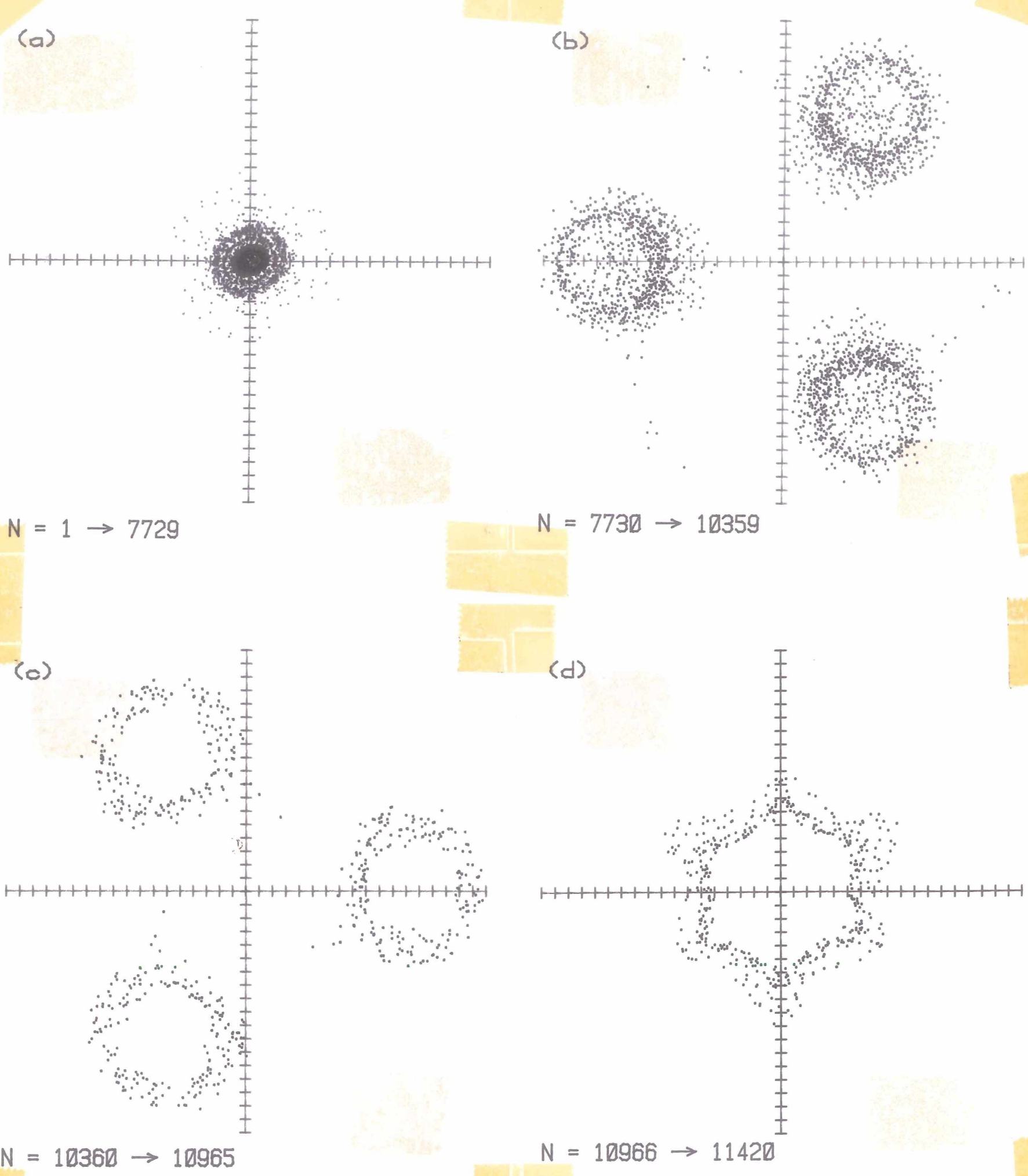

Fig. 4: Particle motion in the phase diagram $z-z^{\prime}$ $\left(Q_{x}=25.2, Q_{z}=23.32, Q_{S}=.07, \xi_{x}=\xi_{z}=.04, \sigma_{x}^{*} / \sigma_{z}^{*}=15\right.$, 4 interaction points, 1 damping time $=3000$ revolutions) 

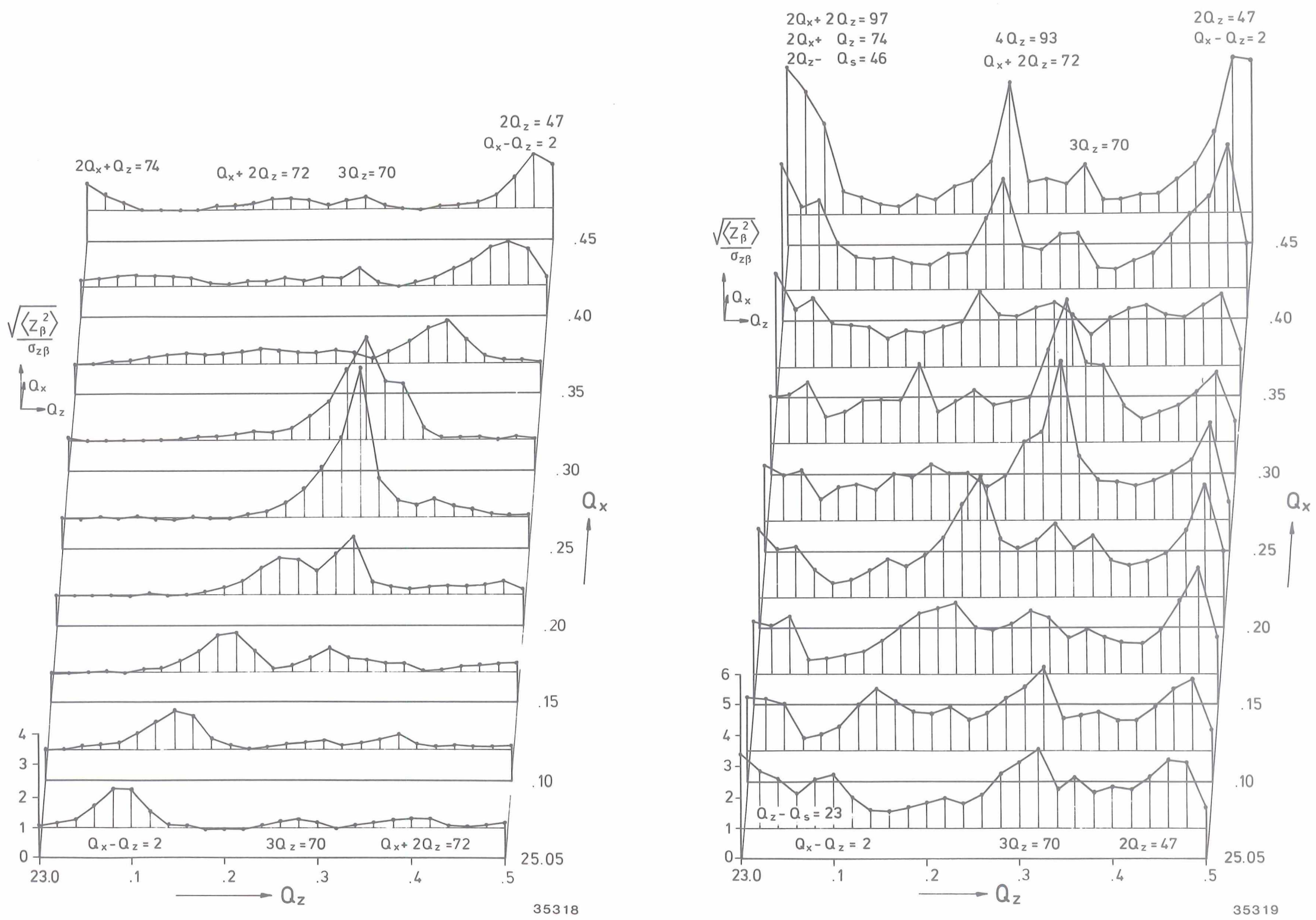

Fig. 5: Increase of beam height as a function of the vertical and horizontal betatron frequency

a) without machine imperfections

b) with machine imperfections $\left(\delta Q_{x, z}= \pm .03, \pm .01\right.$, $\left.D_{x}^{*}= \pm 8.5, \pm 2.8 \mathrm{~cm}, D_{Z}^{*}= \pm 1.3, \pm .4 \mathrm{~cm}\right)$ 
The increase in beam height is given by the root mean square of the vertical betatron coordinates of many particles over many revolutions:

$$
\sqrt{\left\langle z_{B}^{2}\right\rangle}=\sqrt{\frac{1}{N} \sum_{i=1}^{N} z_{B i}^{2}}
$$

$\mathrm{N}$ includes all particles at all interaction points and all revolutions after 4 damping times.

Fig. 5 shows the influence of machine imperfections on the blow-up. To reduce the computer time these simulations were done only for the case "weak-strong", however, simulations of the case "strong-strong" have shown that the dependence on the working point is very similar in both cases. The assumed phase asymmetries can be produced by the usually observed orbit displacements in the sextupoles, and the magnitude of the spurious dispersions at the interaction points is scaled from measurements of the dispersions in the straight sections outside the mini beta insertion.

\section{References}

1. A. Piwinski, A. Wrulich: Excitation of Betatron-Synchrotron Resonances by a Dispersion in the Cavities, DESY 76/07 (1976)

2. N.A. Vinoburow et al.: Synchrobetatron Resonances at Zero Value of Chromaticity, 10th Intern. Conf. on High Energy Accel., Protvino, 254 (1977)

3. R.M. Sundelin: Synchrobetatron Oscillation Driving Mechanism, IEEE Trans. Nucl. Sci. NS-26, 3604 (1979)

4. A. Piwinski: Synchro-Betatron Resonances, llth Intern. Conf. on High Energy Accel., Geneva, 562 (1980)

5. A. Piwinski: Limitation of the Luminosity by Satellite Resonances, DESY 77/18 (1977)

6. H. Nesemann, K. Wille: Operational Experience with DORIS II, 12th Intern. Conf. on High Energy Accel., Fermilab (1983)

7. J. Tennyson, Univ. of Calif., Berkeley (1980) (unpublished)

8. S. Peggs, R. Talman: Observations at CESR and Theory of the Beam-Beam Luminosity Limitation, llth Intern. Conf. on High Energy Accel., Geneva, 754 (1980)

9. A. Piwinski: Computer Simulations of the Beam-Beam Interaction, llth Intern. Conf. on High Energy Accel., Geneva, 751 (1980)

10. A. Piwinski: Computer Simulation of the Beam-Beam Interaction, DESY 80/131 (1980) 